\title{
Constraining Two dimensional CFTs with Modular Invariance
}

\author{
Jin-Beom Bae* \\ Korea Institute for Advanced Study \\ E-mail: jinbeomekias.re.kr
}

We discuss how the modular property constraints partition function of two-dimensional conformal field theories(CFT). We apply the semi-definite programming to analyze the modular constraints. We verified that the modular bootstrap program find the partition of various rational CFTs. This includes the levelone Wess-Zumino-Witten(WZW) models with Degline's exceptional series, cousins of extremal CFTs, babymonster CFT and c=8 and c=16 CFTs without Kac-Moody symmetry. 


\section{INTRODUCTION}

Conformal field theory (CFT) has been played a key role in theoretical physics. It has been highlighted both in the string theory and the statistical mechanics. For the two-dimensional CFTs, the infinite dimensional Virasoro symmetry is known to stronger than the conformal symmetry in higher dimensional $(D>2)$ spacetime. Specifically, the physical quantities, i.e., spectrum, partition function and correlation function, of $c<1$ rational CFTs were completely solved using the crossing symmetry constraints and Virasoro symmetry [1]. However, the full classification of the unitary two-dimensional CFTs is still out of reach. Unlike the minimal models, the modular invariance and crossing symmetry cannot provide enough constraints for $c \geq 1$ CFTs. Nevertheless, recently it has been noticed that the further constraints can be originated from the crossing symmtery, conformal symmetry and unitarity. This method is referred to as conformal bootstrap program, and it provided new insights to the CFTs [2,3].

Inspired from this philosophy, we study the consequences of the modular invariance, focusing on the $c>1$ two-dimensional CFTs [4]. The previous works [5-7] mostly considered the theories without extended chiral algebra. Instead, we mainly focus on the CFTs with holomorphic/anti-holomorphic currents. We verified that the modular bootstrap program successfully find the torus partition function of various twodimensional CFTs. In this article, we will brefly review the basic idea of modular bootstrap program and provide the main results of our previous paper [4].

\section{MODULAR BOOTSTRAP PROGRAM}

In general, the torus partition function of the two-dimensional CFT is defined as

$$
Z(\tau, \bar{\tau})=\operatorname{Tr}\left[e^{-2 \pi \tau_{2} H} e^{2 \pi i \tau_{1} J}\right],
$$

where trace means summing over the states of a given CFT along the circle. One can decompose the partition function (2.1) into the sum over the Virasoro characters as

$$
Z(\tau, \bar{\tau})=\chi_{0}(\tau) \bar{\chi}_{0}(\bar{\tau})+\sum_{h, \bar{h}} d_{h, \bar{h}}\left[\chi_{h}(\tau) \bar{\chi}_{\bar{h}}(\bar{\tau})+\chi_{\bar{h}}(\tau) \bar{\chi}_{h}(\bar{\tau})\right]+\sum_{j=1} d_{j}\left[\chi_{j}(\tau) \bar{\chi}_{0}(\bar{\tau})+\chi_{0}(\tau) \bar{\chi}_{j}(\bar{\tau})\right]
$$

where $\chi_{h}(\tau)$ denotes the Virasoro character of the primary state with conformal weight $(h, \bar{h})$. Also, $d_{h, \bar{h}}$ and $d_{j}$ mean the degeneracies. Precisely, the vacuum and primary Virasoro characters have a form of

$$
\chi_{0}(\tau)=q^{-\frac{c}{24}} \prod_{n=2}^{\infty} \frac{1}{1-q^{n}}, \quad \chi_{h}(\tau)=q^{h-\frac{c}{24}} \prod_{n=1}^{\infty} \frac{1}{1-q^{n}} .
$$

The torus partition function (2.2) should be invariant under the modular transformation $S L(2, \mathbb{Z})$ that is generated by $T$ and $S$,

$$
T: \tau \rightarrow \tau+1, \quad S: \tau \rightarrow-\frac{1}{\tau},
$$

unless a given CFT does not have the large diffeomorphism anomaly. One can easily show that the spin of all states should be integer, due to the requirement of invariance under the $T$-transformation. The constraints of the $S$-invariance is more subtle, the spectrum $d_{h, \bar{h}}$ and $d_{j}$ are forced to satisfy

$$
0=\mathscr{Z}_{\mathrm{vac}}(\tau, \bar{\tau})+\sum_{h, \bar{h}} d_{h, \bar{h}}\left[\mathscr{Z}_{h, \bar{h}}(\tau, \bar{\tau})+\overline{\mathscr{Z}}_{h, \bar{h}}(\tau, \bar{\tau})\right]+\sum_{j=1} d_{j}\left[\mathscr{Z}_{j}(\tau, \bar{\tau})+\overline{\mathscr{Z}}_{j}(\tau, \bar{\tau})\right]
$$


where

$$
\begin{gathered}
\mathscr{Z}_{\mathrm{vac}}(\tau, \bar{\tau})=\chi_{0}(\tau) \bar{\chi}_{0}(\bar{\tau})-\chi_{0}\left(-\frac{1}{\tau}\right) \bar{\chi}_{0}\left(-\frac{1}{\bar{\tau}}\right), \\
\mathscr{Z}_{h, \bar{h}}(\tau, \bar{\tau})=\chi_{h}(\tau) \bar{\chi}_{\bar{h}}(\bar{\tau})-\chi_{h}\left(-\frac{1}{\tau}\right) \bar{\chi}_{\bar{h}}\left(-\frac{1}{\bar{\tau}}\right), \\
\mathscr{Z}_{j}(\tau, \bar{\tau})=\chi_{j}(\tau) \bar{\chi}_{0}(\bar{\tau})-\chi_{j}\left(-\frac{1}{\tau}\right) \bar{\chi}_{0}\left(-\frac{1}{\bar{\tau}}\right) .
\end{gathered}
$$

Now, our goal is to investigate the consequences of the bootstrap equation (2.5) on the spectrum of operators.

It is very difficult to solve the constraint equation (2.5) analytically. This is because there are infinitely many unknown parameters, namely the degeneracies. To circumvent this difficulty, one can employ the numerical method of semi-definite programming (SDP). To apply the SDP, we first make a hypothesis on the CFT spectrum. As a second step, we search for a linear functional $\alpha$ satisfying the conditions

$$
\alpha\left[\mathscr{Z}_{\mathrm{vac}}(\tau, \bar{\tau})\right]=1, \quad \alpha\left[\mathscr{Z}_{h, \bar{h}}(\tau, \bar{\tau})\right] \geq 0, \quad \alpha\left[\mathscr{Z}_{j}(\tau, \bar{\tau})\right] \geq 0 .
$$

If such an $\alpha$ exists, one can conclude that

$$
\alpha\left[\mathscr{Z}_{\mathrm{vac}}(\tau, \bar{\tau})\right]+\sum_{h, \bar{h}} d_{h, \bar{h}} \alpha\left[\mathscr{Z}_{h, \bar{h}}(\tau, \bar{\tau})+\overline{\mathscr{Z}}_{h, \bar{h}}(\tau, \bar{\tau})\right]+\sum_{j=1} d_{j} \alpha\left[\mathscr{Z}_{j}(\tau, \bar{\tau})+\overline{\mathscr{Z}}_{j}(\tau, \bar{\tau})\right]>0
$$

due to the positivity of $d_{h, \bar{h}}$ and $d_{j}$. This means that (2.5) cannot be satisfied, and as a consequence, the corresponding hypothetical CFT is ruled out.

\section{NUMERICAL RESULTS}

To solve the constraint equation (2.8), we take the spin-dependent linear functional of the form

$$
\widetilde{\alpha}[f(\beta, \bar{\beta})]=\left.\sum_{p=0}^{N} \sum_{m+n=2 p+1}\left(\beta \frac{\partial}{\partial \beta}\right)^{m}\left(\bar{\beta} \frac{\partial}{\partial \bar{\beta}}\right)^{n}\right|_{\beta=1, \bar{\beta}=1} .
$$

Then we find the linear functional $\widetilde{\alpha}$ such that satisfy the below constraint.

$$
\widetilde{\alpha}\left[\mathscr{Z}_{\mathrm{vac}}(\beta, \bar{\beta})\right]=1, \quad \widetilde{\alpha}\left[\mathscr{Z}_{\Delta}(\beta, \bar{\beta})\right] \geq 0 \text { for } \Delta \geq \Delta_{t}
$$

Rather than including all the unitary operators in (3.2), one can hypothesize a gap in the spectrum of primaries. Here we only discuss the twist gap problem, namly we consider the universal gap $\Delta_{t}$ on the twist $t=\Delta-j$. Then we solve SDP problem with two free parameters $c$ and $\Delta_{t}$, using the SDPB package [8]. The main numerical results are summarized in the figure 1.

It turns out that the level-one WZW models with affine Lie algebra $\mathfrak{g}=A_{1}, A_{2}, G_{2}, D_{4}, F_{4}, E_{6}, E_{7}, E_{8}$ are realized on the numerical boundary of twist gap problem. They are the simplest examples of $c \geq 1 \mathrm{RCFT}$ with extended chiral algebras. Moreover, it turns out that the $\left(\widehat{E_{8}}\right)_{1} \mathrm{WZW},\left(\widehat{E_{8}} \times \widehat{E_{8}}\right)_{1} \mathrm{WZW}$, Monster CFT, and babymonster CFT are realized on the numerical boundary. Using the extremal functional method, one can determine the torus partition function of above theories.

\section{References}

[1] A.A. Belavin, Alexander M. Polyakov, A.B. Zamolodchikov, Nucl.Phys. B241 (1984) 333-380. 


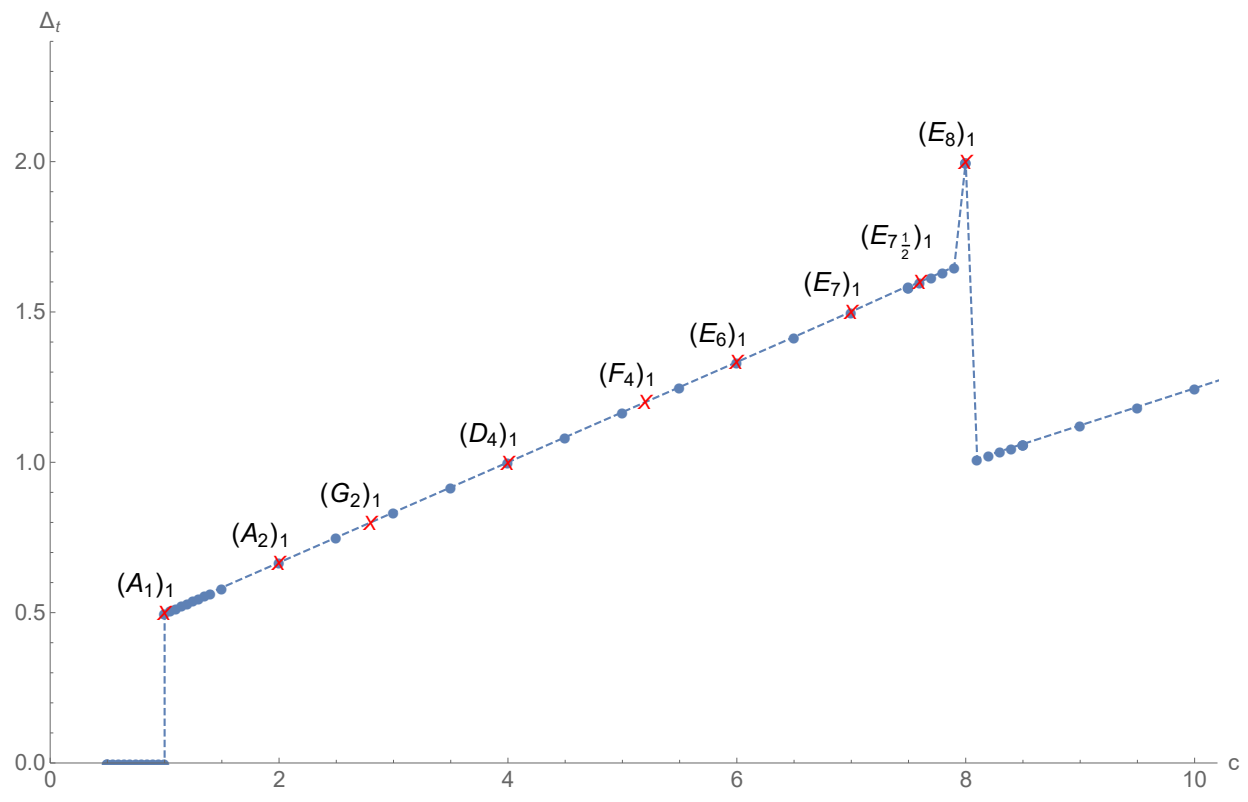

(a) Numerical upper bounds on the twist gap $\Delta_{t}$ in the range of $1 \leq c \leq 10$.

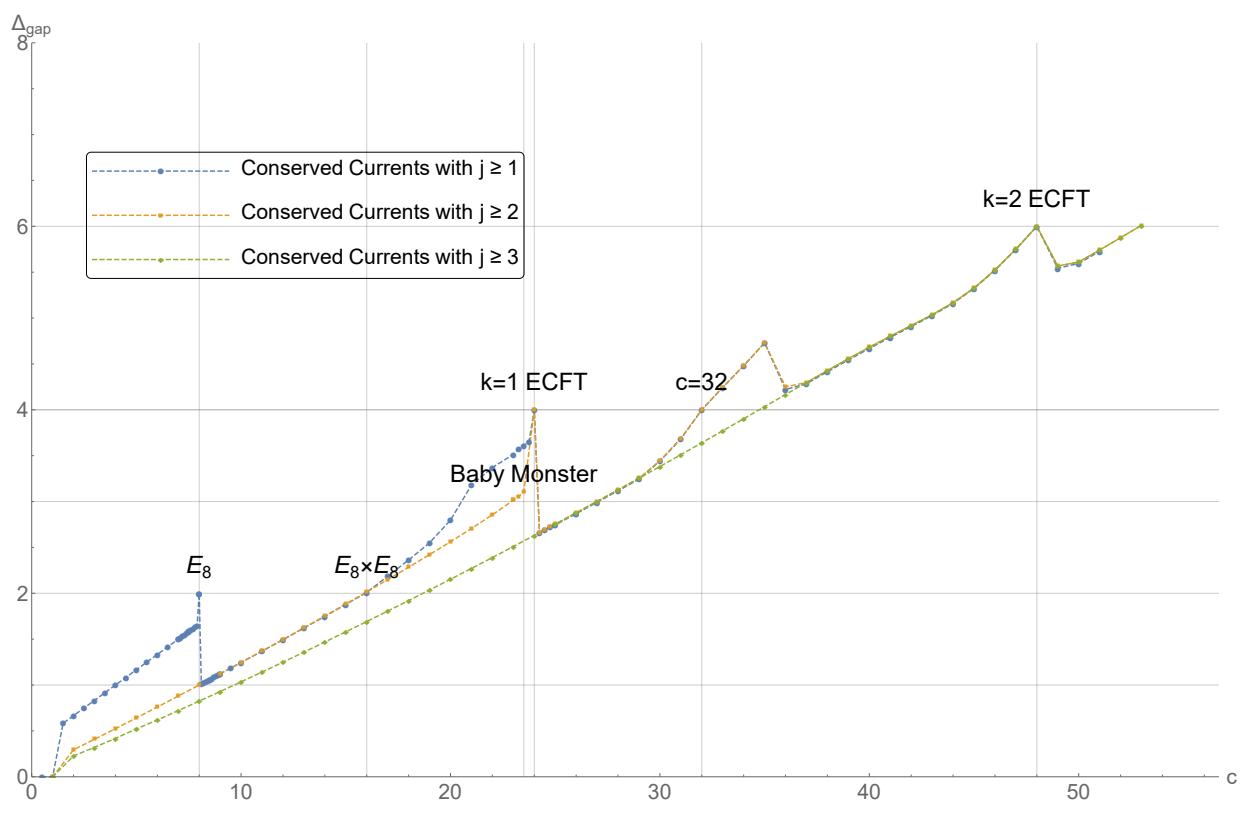

(b) Numerical upper bounds on the twist gap in the range of $1 \leq c \leq 55$ with the conserved currents of $j \geq 1, j \geq 2$ and $j \geq 3$ included.

Figure 1: The numerical bound on the twist gap $\Delta_{t}$.

[2] R. Rattazzi, S. Rychkov, E. Tonni, A. Vichi , JHEP 0812 (2008) 031.

[3] D. Poland, D. Simmons-Duffin, JHEP 1105 (2011) 017.

[4] J. Bae, S. Lee, J. Song, JHEP 1712 (2017) 045.

[5] S. Hellerman, JHEP 1108 (2011) 130.

[6] D. Friedan, C. A. Keller, JHEP 1310 (2013) 180.

[7] S. Collier, Y. Lin, X. Yin, JHEP 1809 (2018) 061.

[8] D. Simmons-Duffin, JHEP 1506 (2015) 174. 